\title{
The periodic variation of 6.7 days for total solar radiation
}

\author{
W. Q. Gan and Y. P. Li \\ Purple Mountain Observatory, Chinese Academy of Sciences, Nanjing 210008, China \\ email: wqgan@pmo.ac.cn
}

\begin{abstract}
Analyzing the power spectrum of Total Solar Irradiance (TSI) for the period from 2003 February 25 to 2009 July 6, observed with the Total Irradiance Monitor (TIM) onboard SOlar Radiation and Climate Experiment (SORCE), we found that there are quite a number of periodic variations. The outstanding shortest one is the period of 6.7 days, about one fourth of the period of solar rotation. Checking the solar magnetic field for the same period of time observed with MDI onboard SOHO, we found that there is about 90 degree difference in longitude for the distribution of solar magnetic field. We therefore conclude that both the 90 degree difference in longitude for the distribution of solar magnetic field and the solar rotation are the reason resulting in the periodic variation of 6.7 days for the total solar radiation.
\end{abstract}

Keywords. Sun: activity, Sun: magnetic fields, Sun: solar-terrestrial relations

\section{Introduction}

The space observation for the Total Solar Irradiance (TSI) with high accuracy began at 1978 (see Fröhlich 2006). It was discovered in the early time that the variation of TSI is related to the disc sunspots and faculae (e.g., Willson et al. 1981). Further studies (e.g., Solanki \& Krivova 2006) show that faculae and bright network made an increase of TSI, while the sunspots let TSI be decreased. The relationships of TSI with some parameters describing the solar activity were studied by many authors (e.g., Minarovjech et al. 2007; Krivova et al. 2007; Tapping et al. 2007) and the TSI was extrapolated backward to the time interval when the observation was unavailable. The connection of TSI with solar magnetic field has also been studied (e.g., Withbroe 2009). In this paper, we use the latest advanced data to study the variation of TSI and try to explain it with a combined effect of solar magnetic field and solar rotation.

\section{Data and Analysis}

The data were taken from the observations with the Total Irradiance Monitor (TIM) instrument onboard SOlar Radiation and Climate Experiment (SORCE) (Kopp et al. 2005). The TSI measurements were provided with a relative standard uncertainty (absolute accuracy) of approximately $0.01 \%$ (100 parts per million, ppm) based on SI units and with a long-term precision (relative accuracy) of $0.001 \% \mathrm{yr}^{-1}(10 \mathrm{ppm})$. The temporal resolution of the data we used is 6 hours. Figure 1 shows the TSI for the period from 2002 February 25 to 2009 July 6, observed at about 1 AU with TIM. We see from the figure that there is a dip drop $(\sim 0.33 \%)$ on 2003 October 29 , when there was a largest solar flare and correspondent big group of sunspots. On average, the TSI is larger at the peak years (when the fluctuation of TSI is also bigger) than at the valley years. 
The power spectrum of TSI is presented in Figure 2. Although there looks quite a number of periods, the obviously shortest one is the period of 6.7 days. This period seems to be related to the discovery of the cutoff of 7 days, based on the data analysis for the initial 153 days, observed with ACRIM onboard SMM (Willson et al. 1981).

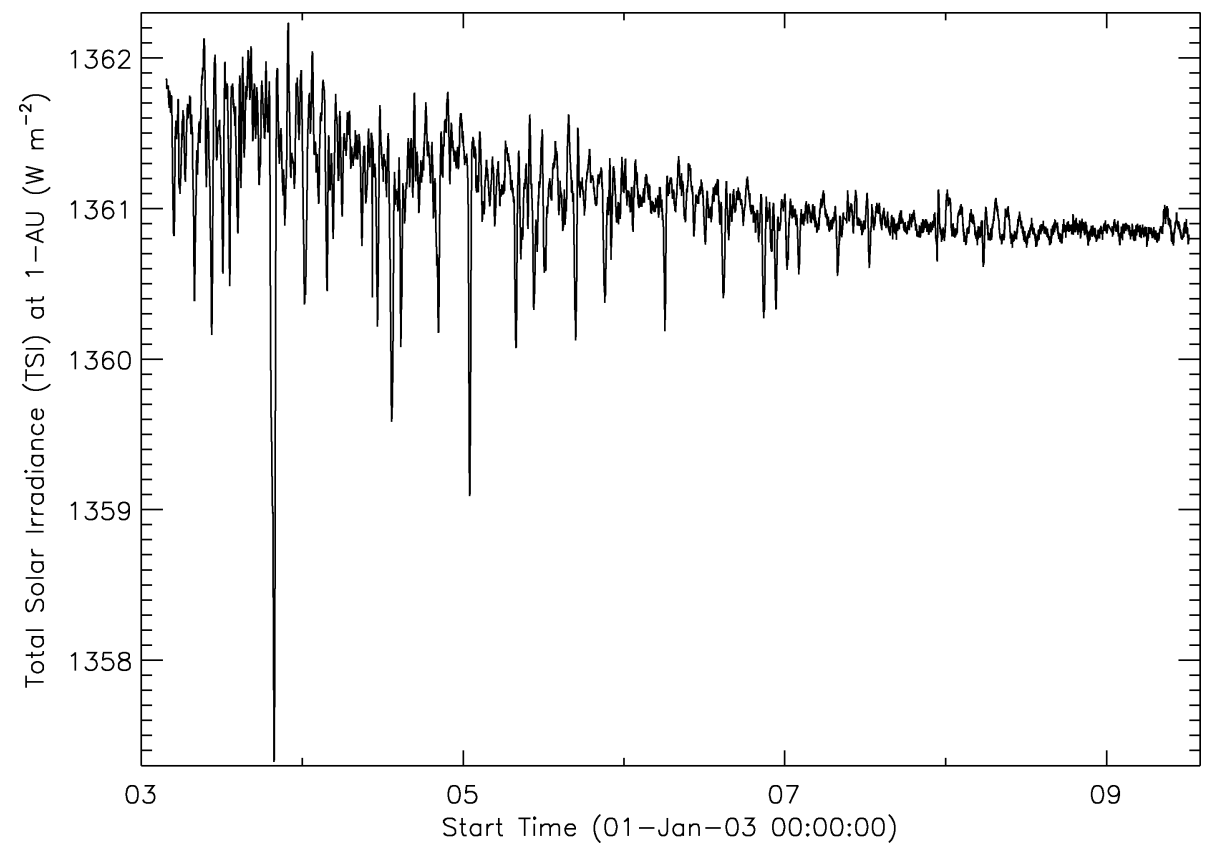

Figure 1. The TSI for the period from 2003 February 25 to 2009 July 6, observed with TIM onboard SORCE.

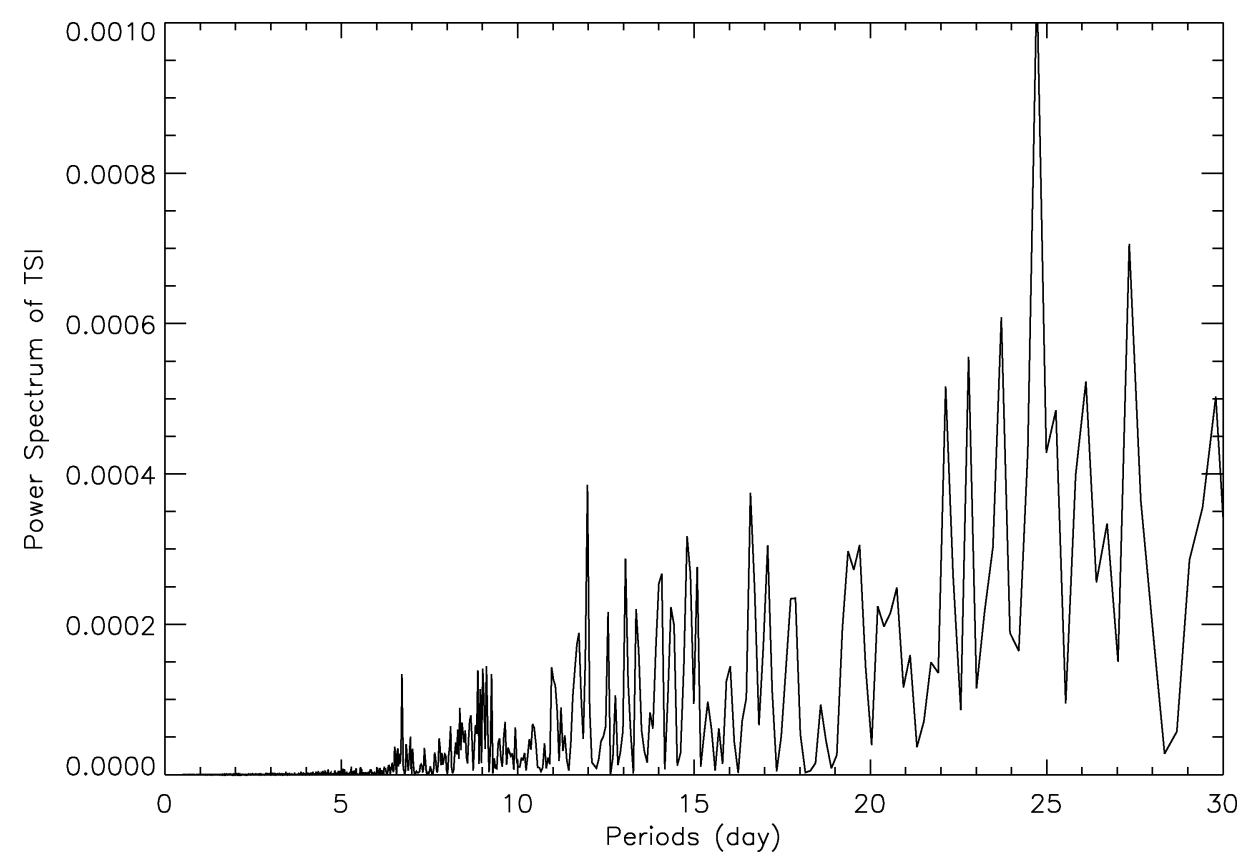

Figure 2. The power spectrum of Figure 1. 
It is so evident that 6.7 days take about one fourth of solar rotational period. Motivated by the relevance of the TSI to the solar magnetic field (e.g., Withbroe 2009), we examined the observations with MDI onboard SOHO. It is indeed found that for some period of time, solar magnetic pattern presents an obvious concentration in the longitude difference of $90^{\circ}$. As an example, Figure 3 shows the magnetic field synoptic chart for the period from 2004 July 22 to 2004 September 14, together with the corresponding TSI. Within two Carrington rotations, the strong magnetic field appears periodically every $90^{\circ}$ in longitude difference. Solar rotation, plus this $90^{\circ}$ distribution of strong magnetic field, provides the most natural explanation on the TSI period of 6.7 days.



Figure 3. Top panel: TSI for the period from 2004 July 22 to 2004 September 14 . Bottom panel: MDI magnetic field synoptic charts.

\section{Summary and Conclusions}

The analysis on TSI shows an obvious period of 6.7 days. We connect this variation to the solar magnetic field. It is concluded that both solar rotation and $90^{\circ}$ longitude difference for the strong magnetic configuration explain naturally the TSI period of 6.7 days.

\section{Acknowledgements}

This work is supported via grants 10773031, 10833007, and 2006CB806302.

\section{References}

Fröhlich, C. 2006, Space Sci. Revs, 125, 53

Kopp, G. \& Lawrence, G. 2005, Solar Phys., 230, 91

Krivova, N. A., Balmaceda, L., \& Solanki, S. K. 2007, A\& A, 467, 335

Minarovjech, M., Rušin, V., \& Saniga, M. 2007, Solar Phys., 241, 269

Solanki, S. K. \& Krivova, N. A. 2006, Space Sci. Revs, 125, 25

Tapping, K. F., Boteler, D., Charbonneau, P., et al. 2007, Solar Phys., 246, 309

Willson, R. C., Gulkis, S., Janssen, M., et al. 1981, Science, 211, 700

Withbroe, G. L. 2009, Solar Phys., 257, 71 